\title{
META-ANALYSIS: THERAPEUTIC EFFECT OF TRANSCATHETER ARTERIAL CHEMOEMBOLIZATION COMBINED WITH COMPOUND KUSHEN INJECTION IN HEPATOCELLULAR CARCINOMA
}

\author{
Qizhe Sun $^{1}$, Wenli Ma ${ }^{1 *}$, Yuan Gao ${ }^{1}$, Wenling Zheng ${ }^{1}$, Bao Zhang ${ }^{1}$, Yifei Peng ${ }^{1}$ \\ ${ }^{1}$ Institute of Genetic Engineering, Southern Medical University,1838 North Guangzhou Road, \\ Guangzhou City, Guangdong Province, 510515, P.R. China. \\ *E-mail: wenlima1964@yahoo.com.cn, wenli668@gmail.com
}

\begin{abstract}
Compound Kushen Injection (CKI) is Sophora flavescens and Heterosmilacis japonicae extract. Meta-analysis confirmed that CKI plus transcatheter arterial chemoembolization (TACE) is more superior to TACE alone for unresectable hepatocellular carcinoma (UHCC) patients.

Key words: Compound Kushen Injection, Transcatheter arterial chemoembolization, TACE, hepatocellular carcinoma The list of abbreviations: $\mathrm{CKI}=$ Compound Kushen Injection; $\mathrm{TACE}=$ transcatheter arterial chemoembolization; $\mathrm{UHCC}=$ unresectable hepatocellular carcinoma; WHO=World Health Organization; SD rat=Sprague Dawley Rat; OR=Odds Ratio; CI=Confidence Interval; KPS=Karnofsky Performance Score.
\end{abstract}

\section{Introduction}

Hepatocellular carcinoma is one of the commonest forms of malignancies. Its global morbidity ranks the seventh, while mortality is at fourth position in the world. Statistics from the World Health Organization (WHO, 2008) show that 749,744 cases of hepatocellular carcinoma were diagnosed, of these, 695,726 cases died in 2008. Southern Africa, Southeast Asia and the Mediterranean coast is the high incidence area of hepatocellular carcinoma. Hepatocellular carcinoma has been the second leading cause of death by cancer in China, there were 372,079 deaths in 2008 (Ferlay et al., 2008). However, due to the spread of hepatitis B, the incidence and mortality of hepatocellular carcinoma also show a rising trend (Liaw et al., $1986)$.

For unresectable hepatocellular carcinoma (UHCC) patients, most of them are at an advanced stage, few meaningful therapeutic options are available (Bruix et al., 2002). TACE is considered as a primary and complementary measure for the treatment of UHCC (Roche et al., 2003; Stuart, 2003; Venook et al., 1990). However, the adverse events of TACE, such as post embolization syndrome, hepatic insufficiency and myelosuppression, are frequent leads to interruption of TACE treatment (Chung et al., 1996). As a result, searching new drugs which can combine with TACE to enhance the therapeutic effects and reduce its adverse events has become a hotspot.

Many clinical trials showed that CKI plus TACE can reduce the adverse reactions and improve quality of life for UHCC (Chen, 2009; Tong, 2010; Zhang et al., 2006; Zhu and Li, 2006). CKI was extracted from the mixture of Sophora flavescens and Heterosmilacis Japonicae with the mass ratio of 7 than 3. Experimental studies confirmed that CKI has significant killing effect on the tumor cells, such as Hep, H22, LAC and Lewis in vitro (Lin et al., 2009). Meanwhile, animal

\footnotetext{
* Corresponding author: Wenli Ma, Institute of Genetic Engineering, Southern Medical University, 1838 North Guangzhou Road, Guangzhou City, Guangdong Province, 510515, P.R. China. Tel.: +86 20 61648210, Fax: +86 20 61647755, E-mail address:wenlima1964@yahoo.com.cn;wenli668@gmail.com.
} http://dx.doi.org/10.4314/ajtcam.v9i2.1 
experiment showed that CKI can depress the growth of hepatocellular carcinoma and its angiogenesis in SD rat (Sprague Dawley Rat) (Li et al., 2007; Zhu et al., 2011). These results revealed that CKI may enhance the therapeutic effects of TACE. Until now, however, rigorously designed, randomized, large, multi-center, double-blind, controlled trials for UHCC have not been reported.

The purpose of this meta-analysis is to evaluate whether CKI enhances therapeutic effects for UHCC after TACE. It is anticipated that this systematic review will provide evidence-based information for clinical practice.

\section{Materials and Methods \\ Criteria for Inclusion and Exclusion}

The articles were read by two reviewers (Qizhe Sun and Yuan Gao) and studies were selected systematically according to the following criteria: (1) hepatocellular carcinoma patients were confirmed cytologically or pathologically, or diagnosed by CT; (2) trials were described as randomized clinical trials (RCTs); (3) published trials included a treatment group receiving CKI plus TACE and a control group receiving TACE; and (4) the published data of primary interest were tumor response and quality of life for calculation of the odds ratio (OR) at a 95\% confidence interval (CI). Trials were excluded if they did not meet the criteria above and included the following: (1) involved animal studies or in vitro studies; (2) did not represent primary research (review articles, letters to the editor, etc); or (3) represented duplicate publications of other studies previously identified in our systematic evaluation.

\section{Literature Search Strategy}

Retrieval of trials was performed through the Cochrane Database of Systematic Reviews (The Cochrane Library, Issue 5, 2011), CENTRAL (the Cochrane Central Register of Controlled Trials, January 1966 to May 2011), MEDLINE (January 1966 to May 2011), EMBASE (January 1966 to May 2011), CNKI (Chinese National Knowledge Infrastructure, January 1979 to May 2011) and CBMdisk (Chinese Biomedical Database, January 1978 to May 2011). All searches were performed without language limitations to identify all relevant trials. The main search terms were: hepatocellular carcinoma, hepatic tumor, hepatic cancer, liver cancer, or liver tumor and transcatheter arterial chemoembolization, TACE transcatheter arterial embolization or TAE and Compound Kushen Injection, Yan Shu, Kushen, Sophora flavescens or Sophorae. The search results were downloaded to a reference database and screened further.

\section{Outcome Measurements}

The main outcome measurements were as follows: (1) Tumor response was evaluated according to the WHO standard for evaluating therapeutic efficacy on solid tumors (Therasse, 2002). Based on the degree of tumor regression, efficacy was evaluated as following: CR (complete response, CT and/or MRI revealed complete clearance of the lesion); PR (partial response, lesion decreased more than 50\%); SD (lesion decreased less than 50\% or increased less than 25\%); PD (size of lesion increased more than $25 \%$ after treatment). Tumor responses were defined as CR+PR. (2) Quality of life was evaluated according to the Karnofsky performance score (KPS) (Yates et al., 1980), which was classified as: Improvement (KPS improved $\geqslant 10$ points after treatment); Stabilization (KPS improved $<10$ points or decreased $<10$ points); Deterioration (KPS decreased $\geqslant 10$ points after treatment). (3) One-year survival. (4) Adverse events were evaluated, based on the WHO criteria for evaluation of acute and subacute toxic and adverse reactions (Miller et al., 1981). 


\section{Review Methods}

\section{Data extraction}

The trials selection and the data extraction were performed independently by two investigators. For conflicts, an agreement was reached by discussion among reviewers. The following information was collected from each study: (1) the information about patients (the number of patients allocated, clinical stage, and KPS); (2) the characteristics of methods (the randomization procedure, concealment of allocation, blinding procedure, withdrawal and reasons, and protection against contamination); (3) The characteristics of interventions (dosage and duration of therapy, TACE course, and any co-interventions; (4) the outcomes (tumor response, quality of life, one-year survival and adverse events).

\section{Quality assessment}

Methodological quality was evaluated according to the Cochrane Handbook for Systematic Reviews of Interventions (Version 5.1.0) (Higgins et al., 2011). The evaluation was performed in the following aspects: (1) selection bias (random sequence generation and allocation concealment); (2) performance bias (blinding of participants and personnel); (3) detection bias (blinding of outcome assessment); (4) attrition bias (incomplete outcome data); (5) reporting bias (selective reporting); (6) other bias (other sources of bias). The judgement of each term was assigned as: 'Low risk' of bias (the description of methods are adequate and the procedures are correct), 'High risk' of bias (the methods or procedures are improper), or 'Unclear risk' of bias (without description of methods and procedures). The methodological quality evaluation was performed independently by two reviewers, and discrepancies were resolved through discussion.

\section{Statistical method.}

Meta-analysis was performed according to recommendations from the Cochrane Handbook for Systematic Reviews of Interventions (Higgins et al., 2011) using the statistical software RevMan5.1.2 (Update Software Ltd, Oxford, England) provided by the Cochrane Collaboration. Statistical heterogeneity was explored using the $x^{2}$ test $(\mathrm{P}<0.10$ was considered representative of significant statistical heterogeneity). The random-effects model was used when there was significant statistical heterogeneity $(\mathrm{P}<0.10)$; otherwise the fixed-effects model was used. For dichotomous variables, OR (Odds Ratio) with $95 \%$ CI was calculated. Patients with incomplete or missing data were counted as treatment failures. The sensitivity analysis was performed by comparing the results of two different statistical models (random-effects model and fixed-effects model). Potential publication bias was examined by funnel plot.

\section{Results}

\section{Common characteristics}

The initial search identified 795 trials for possible inclusion in the review, but all of these trials were reported in Chinese journals. According to the inclusion criteria, 785 trials were excluded as obvious error, non-clinical studies, duplicates, or study objectives different from the aim of this review. Ultimately, 10 trials (Cao et al., 2009; Chen et al., 2006; Chen et al., 2007; Deng et al., 2009; Guan et al., 2006; Huang et al., 2006; Wang and Cheng, 2009; Xu et al., 2010; Yang, 2006; Yu and Kang, 2009) were identified for further study (726 patients), but without a multi-center study. Characteristics of the included trials are listed in Tables 1 and 2. 


\begin{tabular}{|c|c|c|c|c|c|c|c|}
\hline \multirow{2}{*}{$\begin{array}{c}\text { Study } \\
\text { Author (year) }\end{array}$} & \multicolumn{2}{|c|}{ No. Enrolled Patients } & \multirow[t]{2}{*}{ Stage } & \multicolumn{2}{|c|}{$\mathrm{KPS}^{\mathrm{a}}$} & \multirow[t]{2}{*}{ Treatment vs Control } & \multirow[t]{2}{*}{ Outcomes } \\
\hline & Treatment & Control & & Treatment & Control & & \\
\hline Cao et al., 2009 & 30 & 30 & II, III & $\geq 60$ & $\geq 60$ & TACE-Kushen vs TACE & Tumor response, KPS, and adverse events \\
\hline Chen et al., 2006 & 16 & 14 & II, III & $>50$ & $>50$ & TACE-Kushen vs TACE & Tumor response, KPS, and adverse events \\
\hline Chen et al., 2007 & 46 & 40 & II, III & $\geq 70$ & $\geq 70$ & TACE-Kushen vs TACE & Tumor response, KPS, survival, and adverse events \\
\hline Deng et al., 2009 & 20 & 20 & II, III & $\geq 70$ & $\geq 70$ & TACE-Kushen vs TACE & Tumor response, KPS, and adverse events \\
\hline Guan et al., 2006 & 58 & 59 & II, III & $>50$ & $>50$ & TACE-Kushen vs TACE & Tumor response, KPS, survival, and adverse events \\
\hline Huang et al., 2006 & 35 & 33 & II, III & $\geq 50$ & $\geq 50$ & TACE-Kushen vs TACE & Tumor response, KPS, and adverse events \\
\hline Wang et al., 2009 & 27 & 30 & II, III & $\geq 70$ & $\geq 70$ & TACE - Kushen vs TACE & Tumor response, KPS, and survival \\
\hline Xu et al., 2010 & 53 & 53 & II, III & - & - & TACE-Kushen vs TACE & Tumor response, KPS, survival, and adverse events \\
\hline Yang, 2006 & 33 & 33 & II, III & $\geq 60$ & $\geq 60$ & TACE-Kushen vs TACE & Tumor response, KPS, and survival \\
\hline Yu et al., 2010 & 48 & 48 & II, III & $>60$ & $>60$ & TACE-Kushen vs TACE & Tumor response, KPS, and adverse events \\
\hline
\end{tabular}

${ }^{\mathrm{a} K P S}$, Karnofsky Performance Scale; ${ }^{b} \mathrm{TACE}$, transcatheter arterial chemoembolization; 
Sun et al., Afr J Tradit Complement Altern Med. (2012) 9(2):178-188

Table 2: Compound Kushen Injection and Transcatheter Arterial Chemoembolization Drugs Used in Included Studies

\begin{tabular}{|c|c|c|c|c|c|c|c|}
\hline \multirow{2}{*}{$\begin{array}{c}\text { Study } \\
\text { Author (year) }\end{array}$} & \multirow[t]{2}{*}{ Anticancer Drug } & \multicolumn{3}{|c|}{ Embolizing } & \multirow{2}{*}{$\begin{array}{c}\text { Duration } \\
\text { Days }\end{array}$} & \multirow{2}{*}{$\begin{array}{c}\text { Compound Kushen Injection } \\
\text { Dosage }\end{array}$} & \multirow{2}{*}{$\begin{array}{l}\text { Injection } \\
\text { Method }\end{array}$} \\
\hline & & LP & Agents & Adjuvant Drug & & & \\
\hline Cao et al., 2009 & 5-Fu, DDP, MMC & Yes & GS particles & MMC & $56<\mathrm{D}<224$ & $\begin{array}{l}\text { given } 20 \mathrm{ml} \text { per time, once daily over } 14 \text { days; began in the TACE operation day; } \\
\text {, }\end{array}$ & I.v.drip \\
\hline Chen et al., 2006 & 5-Fu, DDP, EPI, & $\mathrm{Na}$ & $\mathrm{Na}$ & $\mathrm{Na}$ & 63 & given $20 \mathrm{ml}$ per time, once daily over 21 days; begin in the TACE operation day; & I.v.drip \\
\hline Chen et al., 2007 & 5-Fu, MMC, EPI, & Yes & $\mathrm{Na}$ & $\mathrm{Na}$ & $84<\mathrm{D}<126$ & given $20 \mathrm{ml}$ per time, once daily over the whole duration; & I.v.drip \\
\hline Deng et al., 2009 & THP-ADM & Yes & $\mathrm{Na}$ & $\mathrm{Na}$ & 61 & given $20 \mathrm{ml}$ per time, once daily over 14 days; begin in the 2 nd day after TACE operation; & I.v.c. infusion \\
\hline Guan et al., 2006 & (5-Fu, DDP, ADM) or (THP-ADM,5-Fu, CBP) & $\mathrm{Na}$ & $\mathrm{Na}$ & $\mathrm{Na}$ & $45<\mathrm{D}<60$ & given $20 \mathrm{ml}$ per time, once daily over the whole duration; & I.v.drip \\
\hline Huang et al., 2006 & 5-Fu, ADM, HCPT & Yes & $\mathrm{Na}$ & $\mathrm{Na}$ & $56<\mathrm{D}<224$ & given $15 \sim 30 \mathrm{ml}$ per time, once daily over $10 \sim 20$ days; begin in the TACE operation day; & I.v.drip \\
\hline Wang et al., 2009 & 5-Fu, DDP, ADM & Yes & GS particles & $\mathrm{Na}$ & $140<\mathrm{D}<168$ & given $20 \mathrm{ml}$ per time, once daily over 10 days; begin in the TACE operation day; & I.v.drip \\
\hline Xu et al., 2010 & 5-Fu, DDP,CF & Yes & $\mathrm{Na}$ & THP-ADM or HCPT & $60<\mathrm{D}<120$ & given $20 \mathrm{ml}$ per time, once daily over 15 days; begin in the 3 rd day before TACE operation; & I.v.drip \\
\hline Yang, 2006 & DDP, MMC, ADM & Yes & GS particles & $\mathrm{Na}$ & $40<\mathrm{D}<240$ & given $20 \mathrm{ml}$ per time, once daily over 20 days; began in the TACE operation day; & I.v.drip \\
\hline Yu et al., 2010 & 5-Fu, ADM, НСРT & Yes & GS particles & $\mathrm{Na}$ & 84 & given $20 \mathrm{ml}$ per time, once daily over 15 days; begin in the TACE operation day; & I.v.drip \\
\hline
\end{tabular}

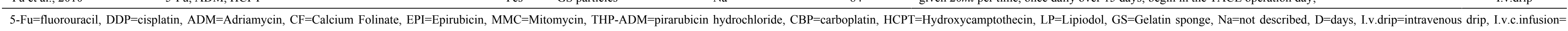
intravenous continuous infusion. 
In terms of quality, all trials mentioned "randomization," but none stated the generation of a random allocation sequence. No trials described information on allocation concealment and blinding. No trials reported withdrawals and dropouts. (Figure 1)

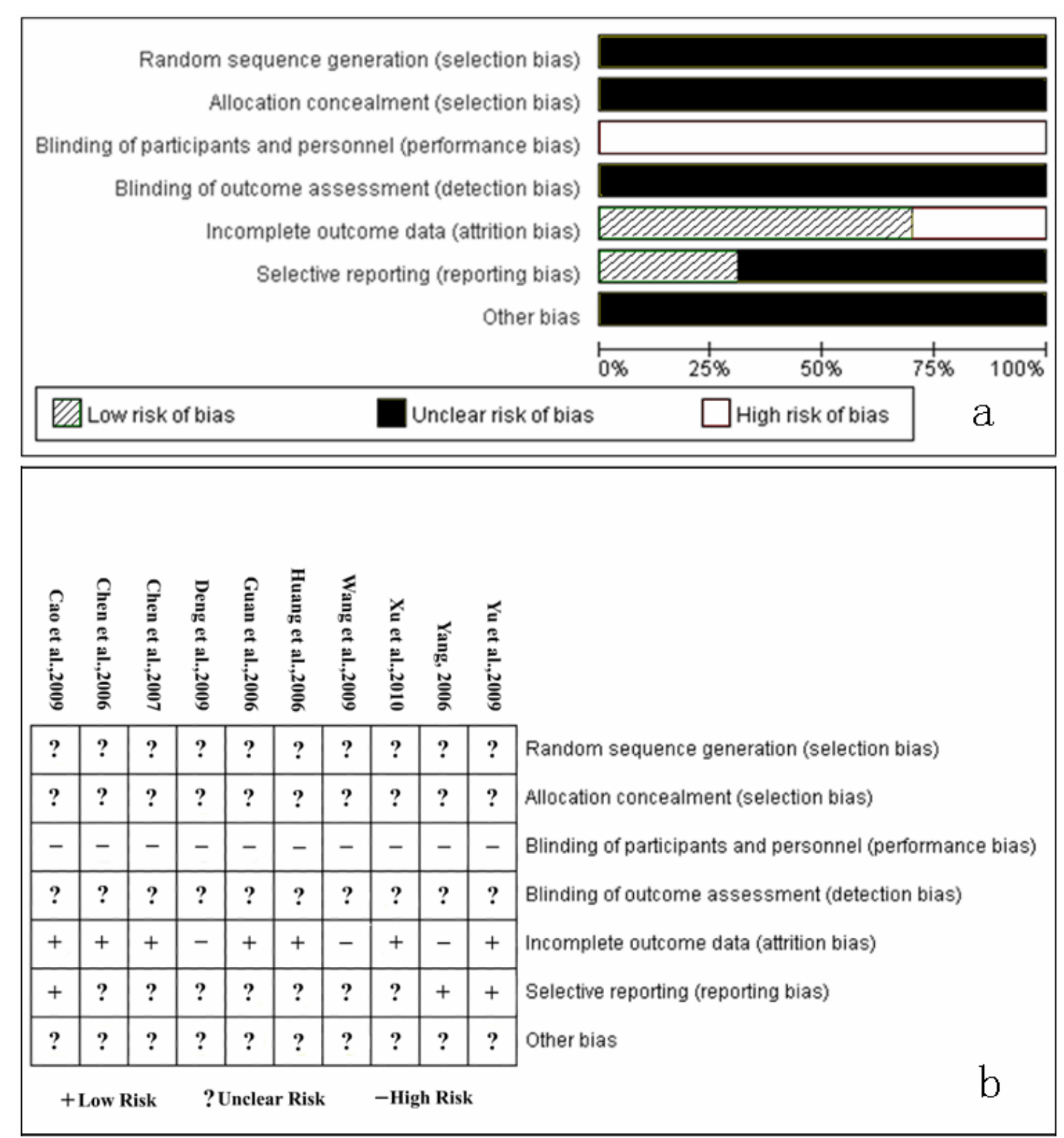

Figure 1: Quality of all included trials a. Risk of bias percentage graph of all included studies b. Risk of bias summary of all included studies

\section{Meta-analysis Outcomes}

\section{Tumor response}

Ten trials (726 patients) (Cao et al., 2009; Chen et al., 2006; Chen et al., 2007; Deng et al., 2009; Guan et al., 2006; Huang et al., 2006; Wang and Cheng, 2009; Xu et al., 2010; Yang, 2006; Yu and Kang, 2009) were identified with a $\mathrm{CR}+\mathrm{PR}$ outcome measurement of tumor response. The fixed-effects model was used because of heterogeneity of the results of trials (Heterogeneity: $\mathrm{Chi}^{2}=4.74$, df $=9(\mathrm{P}=0.86) ; \mathrm{I}^{2}=0 \%$ ). The pooled analysis showed that compared with TACE alone, CKI plus TACE significantly improved the tumor response $(\mathrm{OR}=2.01$ 
$95 \%$ CI [1.47, 2.74]; $\mathrm{P}<0.0001$ ) (Figure 2).

\begin{tabular}{|c|c|c|c|c|c|c|c|c|c|c|}
\hline \multirow[b]{2}{*}{ Studv or Subgroup } & \multicolumn{2}{|c|}{ C.K.I.+TACE } & \multicolumn{2}{|c|}{ TACE } & \multirow{2}{*}{ Weight } & \multirow{2}{*}{$\begin{array}{c}\text { Odds Ratio } \\
\text { M-H, Fixed, 95\% } \mathrm{Cl}\end{array}$} & \multirow{2}{*}{\multicolumn{4}{|c|}{$\begin{array}{c}\text { Odds Ratio } \\
\text { M-H, Fixed, 95\% } \mathrm{Cl}\end{array}$}} \\
\hline & & Total & Events & Total & & & & & & \\
\hline Cao et al., 2009 & 17 & 30 & 12 & 30 & $9.3 \%$ & $1.96[0.70,5.48]$ & & & $\rightarrow-$ & \\
\hline Chen et al., 2006 & 6 & 16 & 3 & 14 & $3.6 \%$ & $2.20[0.43,11.22]$ & & & $\rightarrow$ & \\
\hline Chen et al., 2007 & 25 & 46 & 19 & 40 & $16.6 \%$ & $1.32[0.56,3.08]$ & & & - & \\
\hline Deng et al., 2009 & 2 & 20 & 1 & 20 & $1.6 \%$ & $2.11[0.18,25.35]$ & & & & \\
\hline Guan et al., 2006 & 34 & 58 & 29 & 59 & $21.2 \%$ & $1.47[0.71,3.04]$ & & & - & \\
\hline Huang et al., 2006 & 12 & 35 & 9 & 33 & $10.9 \%$ & $1.39[0.49,3.92]$ & & & - & \\
\hline Wang et al., 2009 & 21 & 27 & 19 & 30 & $7.1 \%$ & $2.03[0.63,6.54]$ & & & 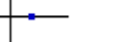 & \\
\hline Xu et al., 2010 & 37 & 53 & 22 & 53 & $11.9 \%$ & $3.26[1.46,7.26]$ & & & $\rightarrow$ & \\
\hline Yang, 2006 & 26 & 33 & 17 & 33 & $6.4 \%$ & $3.50[1.19,10.28]$ & & & & \\
\hline Yu et al., 2009 & 37 & 48 & 28 & 48 & $11.5 \%$ & $2.40[0.99,5.82]$ & & & $=$ & \\
\hline Total $(95 \% \mathrm{Cl})$ & & 366 & & 360 & $100.0 \%$ & $2.01[1.47,2.74]$ & & & $\bullet$ & \\
\hline Total events & 217 & & 159 & & & & & & & \\
\hline $\begin{array}{l}\text { Heterogeneity: } \mathrm{Chi}^{2} \\
\text { Test for overall effec }\end{array}$ & $\begin{array}{l}4.74, d f= \\
Z=4.37\end{array}$ & $\begin{array}{l}9(P=0 \\
P<0.00\end{array}$ & 86); $\left.\right|^{2}=$ & & & & $\begin{array}{r}0.001 \\
\text { C.K }\end{array}$ & $\begin{array}{c}0.1 \\
\text { CII. + TACE }\end{array}$ & $\begin{array}{l}1 \quad 10 \\
\text { TACE }\end{array}$ & 1000 \\
\hline
\end{tabular}

Figure 2: Meta-analysis of tumor response. Compound Kushen Injection (C.K.I.) plus transcatheter arterial chemoembolization (TACE) versus TACE alone for tumor response (CR+PR) of patients with unresectable hepatocellular carcinoma.

Quality of life

There were 9 trials (Cao et al., 2009; Chen et al., 2006; Chen et al., 2007; Guan et al., 2006; Huang et al., 2006; Wang and Cheng, 2009; Xu et al., 2010; Yang, 2006; Yu and Kang, 2009) which contained a KPS improvement of $>10$ points. The fixed-effects model was used (Heterogeneity: $\left.\mathrm{Chi}^{2}=5.51, \mathrm{df}=8(\mathrm{P}=0.70) ; \mathrm{I}^{2}=0 \%\right)$. The results showed that CKI plus TACE significantly improved KPS (OR=2.47; 95\% CI [1.77, 3.44]; P < 0.00001) (Figure 3).

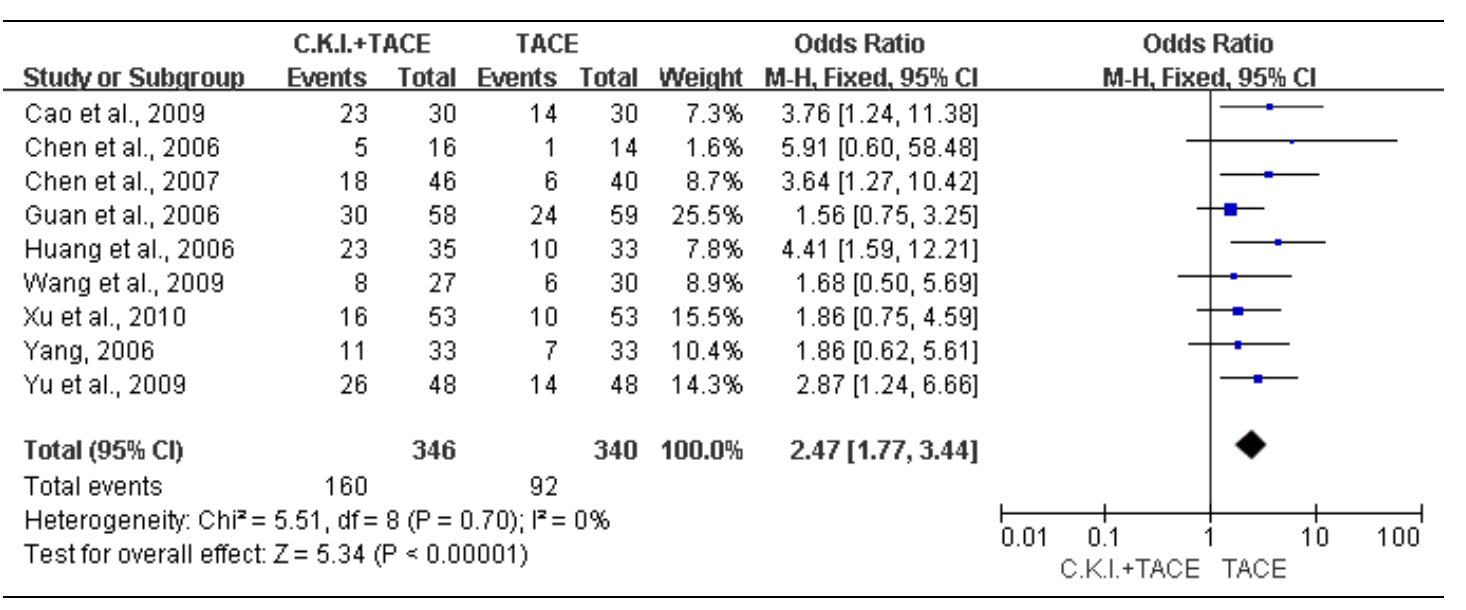

Figure 3: Meta-analysis of quality of life improvement. Compound Kushen Injection (C.K.I.) plus transcatheter arterial chemoembolization (TACE) versus TACE alone for KPS ( $>10$ points) of patients with unresectable hepatocellular carcinoma.

One-year survival

Five trials (432 patients) (Chen et al., 2007; Guan et al., 2006; Wang and Cheng, 2009; Xu et al., 2010; Yang, 2006;) were identified with the outcome measurements of one-year survival. The fixed-effects model was 
used (Heterogeneity: $\left.\mathrm{Chi}^{2}=1.92, \mathrm{df}=4(\mathrm{P}=0.75) ; \mathrm{I}^{2}=0 \%\right)$. The meta-analysis showed that $\mathrm{CKI}$ plus TACE significantly improved one-year survival ( $\mathrm{OR}=2.16$; 95\% CI [1.45, 3.23]; $\mathrm{P}=0.0002)$ (Figure 4).

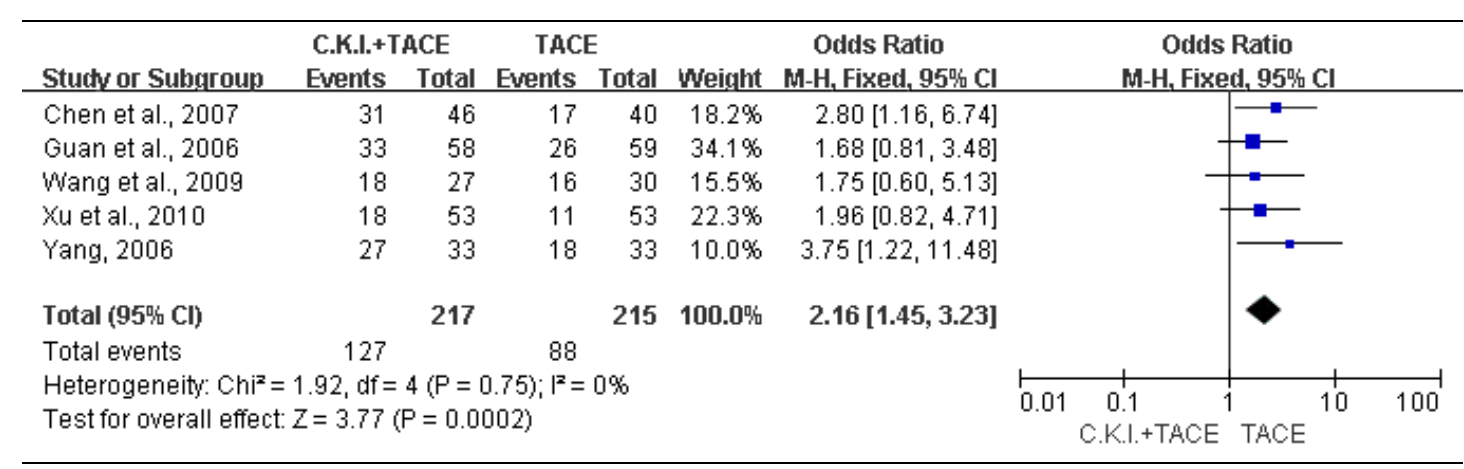

Figure 4: Meta-analysis of one-year survival. Compound Kushen Injection (C.K.I.) plus transcatheter arteria chemoembolization (TACE) versus TACE alone for one-year survival of patients with unresectable hepatocellular carcinoma.

\section{Adverse Effects}

We found 8 trials (Cao et al., 2009; Chen et al., 2006; Chen et al., 2007; Deng et al., 2009; Guan et al., 2006; Huang et al., 2006; Xu et al., 2010; Yu and Kang, 2009) describing adverse events. All of the eight trials reported that the common symptoms such as abdominal pain, abdominal distension, diarrhea, hepatic insufficiency, and bone marrow depression were found in both groups. These might be due to the complications of TACE. There were six trials (Cao et al., 2009; Chen et al., 2006; Chen et al., 2007; Huang et al., 2006; Xu et al., 2010; Yu and Kang, 2009) which showed that compared with TACE alone, CKI plus TACE significantly reduced the complications of TACE. In addition, two trials (Deng et al., 2009; Guan et al., 2006) reported that no adverse events attributable to CKI were observed. Therefore, we concluded that the use of CKI has no significant adverse events.

Sensitivity Analysis

Sensitivity analysis was performed by comparing fixed-effects versus random- effects. In the primary analysis, outcome on quality of life was applied to the fixed-effects model (OR=2.47; 95\% CI $[1.77,3.44]$; $\mathrm{P}<$ $0.00001)$. Therefore, a random-effects model was used to re-analyzed it $(\mathrm{OR}=2.45 ; 95 \% \mathrm{CI}[1.75,3.43] ; \mathrm{P}<$ $0.00001)$. The results were virtually identical.

\section{Publication Bias}

We used the funnel plot to access the publication bias of literatures (Figure 5). The shape of the funnel plots seemed symmetric, and suggesting there was no obvious publication bias.

\section{Discussion}

Experimental studies Show CKI can significantly enhance the apoptosis rates of HepG2 cells and inhibit its transition from G2 phase to M phase in vitro. Therefore, the proliferation of tumor cells, such as HepG2 
cells, BEL-7402 cells and SGC-7901 cells could be restrained by CKI. Further Studies show that the expression of tumor metastasis suppressor gene-nm23 could remarkably be promoted by CKI in BEL-7402 cells, meanwhile in contrast, the expression of $\mathrm{CD}_{44 \mathrm{v} 6}$ in BEL-7402 cells could be depressed (Li et al., 2006). Based on these data, CKI could be a complementary drug with TACE to inhibit the growth of liver cancer, suppress the tumor metastasis, and improve quality of patients' life for UHCC.

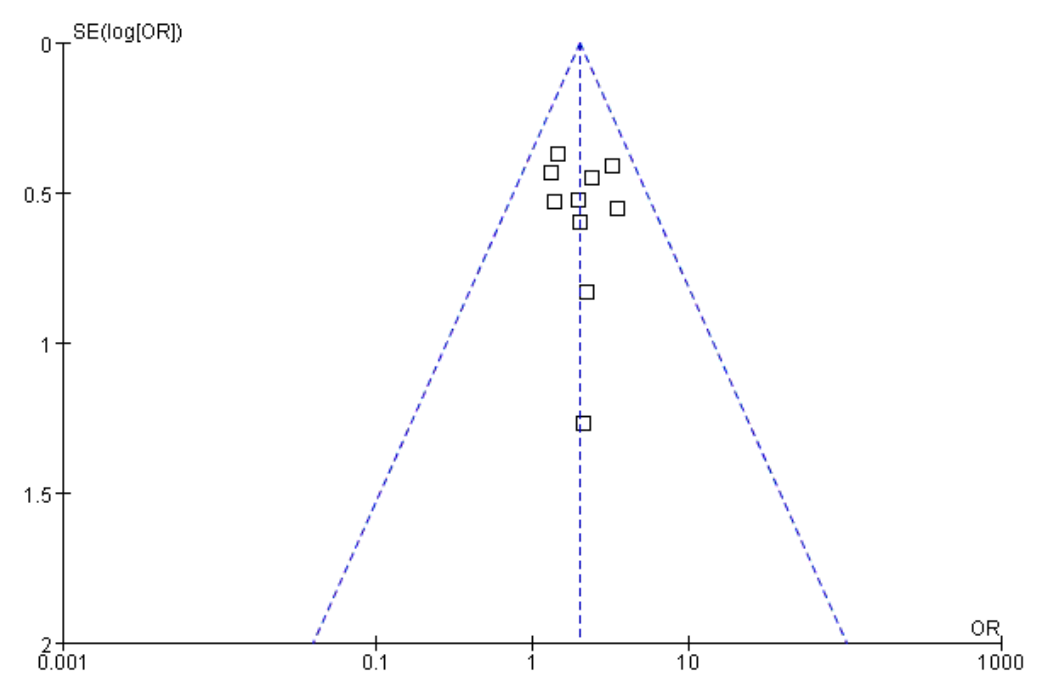

Figure 5: Funnel plot of tumor response (CR+PR). A graphical display of the logarithm of odds ratio (OR) plotted against OR. The vertical line indicates that there was no publication bias.

The results show that TACE plus CKI seemed superior to TACE alone for UHCC in respect to patients' tumor response, quality of life and one-year survival. Although the quality of included literature are low, the results of this study present credible evidence that the administration of CKI plus TACE is worthy of additional study. Hence, larger, longer-term, rigorously designed, multi-center, randomized, double-blind, controlled trials are required to fully assess whether CKI plus TACE is more outstanding than TACE alone.

There are some limitations in this meta-analysis. Due to all literature we found were of poor methodological quality, definite conclusions based on our data could not be made. All included trials mentioned "randomized," but all of them did not describe the information of randomization sequence generation, allocation concealment, and blinding. All included literatures did not report withdrawals and dropouts. We found that the number of patients in experimental group and control group has no change before and after treatment. So it is possible that there are no withdrawals and dropouts. Therefore, the evaluation of one-year survival should be interpreted with caution

\section{Acknowledgments}

We thank the anonymous referee for his/her helpful comments, which remarkably improved the quality of this paper.

\section{References}

1. Bruix, J. and J. M. Llovet (2002). Prognostic prediction and treatment strategy in hepatocellular carcinoma. Hepatology.35(3): 519-524 
2. Cao, J., Wang, Z. L., Fang, J., Liu, H. Q., He, Y. (2009). Clinical Study of Compound Kushen Injection Combined with TACE for Treatment of Advanced Liver Cancer. Shandong Medicine. 49(4): 74-76.

3. Chen, G. H., Li, S.R., Yang, L., (2007). Therapeutic effects of complex kushen injection combined with double interventional therapy on treating primary hepatocarcinoma. Chin J Int egr T rad West Med Dig. 15(4) 239-241.

4. Chen, Y. H. (2009). Efficacy of transcatheter arterial chemoembolization combined with complex prescription kushen injection in the treatment of primary advanced liver cancer. China Modern Medicine. 16(17): 5-6.

5. Chen, Y. L., Jia, Y. J., Zhang, Y., (2006). Clincial effect Observation of Complex Sophorae Injection Combining with Chemotherapy on Treating Liver Cancer. Journal of Tianjin University of Traditional Chinese Medicine. 25(3): 166-167.

6. Chung, J. W., Park, J. H., Han, J. K., Choi, B. I., Han, M. C., Lee, H. S., Kim, C. Y. (1996). Hepatic tumors predisposing factors for complications of transcatheter oily chemoembolization. Radiology. 198(1): 33-40.

7. Deng, L., Li, Z. W., Wu, H. T., Lu, Q. H., Chen, L. Z., Wu, J. Y., Wu, X. Y., Chen, Q. Q., (2009). Therapeutic Effect of Continuous Infusion of Compound Kushen Injection Combined with Hepatic Arterial Chemoembolization for Advanced Liver Cancer: An Observation of 20 Cases. Journal of New Chinese Medicine. 41(4): 28-29

8. Ferlay, J., Shin, H.R., Bray, F., (2008). GLOBOCAN 2008, Cancer incidence and mortality worldwide: IARC CancerBase [DB/OL]. http://globocan.iarc.fr.

9. Guan, C. N., Cai, L.Z., Yue, L. Q., Zhang, Y., (2006). Clinicel study on treatment of advanced primary liver cancer by Yanshu injection combining with chemotherapy. China Journal of Chinese Materia Medica. 31(6): 510-512.

10. Higgins, JPT, Green, S.,(editors) (2011). Cochrane Handbook for Systematic Reviews of Interventions Version 5.1.0 [updated March 2011]. The Cochrane Collaboration. Available from http://www.cochrane-handbook.org.

11. Huang, R. W., Chen, S. X., Huang, X. P., (2006). Clinical Observation On Advanced Primary Hepatic Carcinoma Treated by Yanshu Injection Combined with Transcatheter Arterial Chemoembolization. Hebei Medicine. 12(5): 443-445

12. Liaw, Y. F., Tai, D. I., Chu, C. M., Lin, D. Y., Sheen, I. S., Chen, T. J., Pao, C. C. (1986). Early detection of hepatocellular carcinoma in patients with chronic type B hepatitis. A prospective study. Gastroenterology. 90(2): 263-267.

13. .Li, M., Qian, X.P., Liu, B. R., (2007). Experimental Study of Composite Radix Sophora Flavescentis Injection with Thermotherapy Inhibiting Angiogenesis. Journal of Clinical Medicine in Practice. 11(3): 57-61.

14. .Li, R., Du, J.P., Hou, Y. S., Chen, Z. T., (2006). Experimental study of Yanshu injection acted on SGC-7901, HepG2 and BEL-7402 tumor cell. Cancer Research and Clinic. 18(1): 8-10.

15. Lin, L. Zhou, D. H., Chen, Y., Liu, Q. H., Chen, X. X., (2009). Experimental Study of Anti - tumor Effect of Compound Radix Sophorae Flavescentis Injection on Lung Cancer Cells and Hepatic Carcinoma Cells. Traditional Chinese Drug Research \& Clinical Pharmacology. 20(1): 21-23.

16. Miller, A. B., Hoogstraten, B., Staquet, M., Winkler, A., (1981). Reporting results of cancer treatment. Cancer. 47(1): 207-214

17. Roche, A., Girish, B. V., de Baere, T., Baudin, E., Boige, V., Elias, D., Lasser, P., Schlumberger, M., Ducreux, M., (2003). Trans-catheter arterial chemoembolization as first-line treatment for hepatic metastases from endocrine tumors. Eur Radiol. 13(1): 136-140.

18. Stuart, K., (2003). Chemoembolization in the management of liver tumors. Oncologist. 8(5): 425-437.

19. Therasse, P., (2002). Evaluation of response: new and standard criteria. Ann Oncol. 13 Suppl 4: 127-129. 
20. Tong, H. Z., (2010). The observation of the clinical effects of transcatheter arterial chemoembolization and complex prescrption kuseng injection on the advanced period of liver cancer. Jiangxi Medical Journal. 45(7): 637-639.

21. Venook, A. P., Stagg, R. J., Lewis, B. J., Chase, J. L., Ring, E. J., Maroney, T. P., Hohn, D. C., (1990) Chemoembolization for hepatocellular carcinoma. J Clin Oncol. 8(6): 1108-1114.

22. Wang, H. M., Cheng, X. M. (2009). Composite Kushen injection combined with hepatic artery embolism on unresectable primary liver cancer. Modern Journal of Integrated Traditional Chinese and Western Medicine. 18(12): 1334-1335.

23. WHO (2008). GLOBOCAN 2008. Cancer Incidence and Mortality Worldwide in 2008 . http://globocan.iarc.fr/factsheets/populations/factsheet.asp?uno=900.

24. Xu, P., Wang, W.D., Lu, J., Fan, C., (2010). Efficacy of Compound Kushen Injection in the Prevention and Treatment of the Adverse Reactions Following Transcatheter Hepatic Arterial Chemoembolization in Patient with Liver Cancer. Evaluation and analysis of drug-use in hospitals of China. 10(5): 457-458.

25. Yang, S. M., (2006). Yan Shu Injecdtion Combined with Chemotherapy on Treating advanced primary liver cancer. Medicine Industry Information. 3(17): 209-210.

26. Yates, J. W., Chalmer, B., McKegney, F. P., (1980). Evaluation of patients with advanced cancer using the Karnofsky performance status. Cancer. 45(8): 2220-2224.

27. Yu, M. L., Kang, X.H., (2009). Clinical Study of Compound Kushen Injection Combined with Hepatic Arterial Chemoembolization for Primary Liver Cancer. Guide of China Medicine. 8(7): 123-125.

28. Zhang, A. M., Wang, W. M., Wu, J. J., (2006). Effect of Yanshu on Primary Hepatic Carcinoma Treated with Interventional Therapy. Evaluation and analysis of drug-use in hospitals of China. 6(6): 374-375.

29. Zhu, X. F., Li, G. A., (2006). Study of Yanshu injection combined with chemoembolization on middle and advanced stage liver cancer. Modern Journal of Integrated Traditional Chinese and Western Medicine. 15(4): 431-432.

30. Zhu, Y. Z., Kong, X.B., Yan, L., (2011). Effect of compound radix sophorae flavescentis injection on Hedgehog signaling pathway activation in rat hepatocarcinoma models. Journal of Clinical Rehabilitative Tissue Engineering Research. 15(2): 257-260. 